\title{
Depression and care-dependency in Parkinson's disease: Results from a nationwide study of 1449 outpatients
}

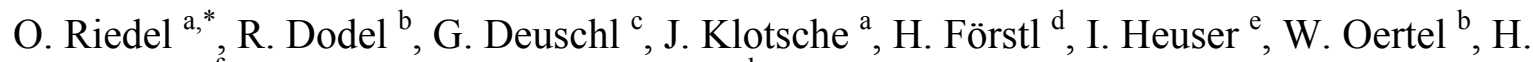 \\ Reichmann ${ }^{\mathrm{f}}$, P. Riederer ${ }^{\mathrm{g}}$, C. Trenkwalder ${ }^{\mathrm{h}}$, H.-U. Wittchen ${ }^{\mathrm{a}}$ \\ a Technische Universitaet Dresden, Institute of Clinical Psychology and Psychotherapy, Chemnitzer Strasse 46, \\ 01187 Dresden, Germany \\ b Phillips-University Marburg, Department of Neurology, Germany \\ c Christian-Albrechts-University Kiel, Department of Neurology, Germany \\ d Technical University of Munich, Department of Psychiatry and Psychotherapy, Germany \\ e Charité-Universitaetsmedizin, Berlin, Germany \\ f Faculty of Medicine Carl Gustav Carus Dresden, Department of Neurology, Germany \\ g University Clinics, Clinic and Polyclinic for Psychiatry, Psychosomatics and Psychotherapy, Wuerzburg, \\ Germany \\ h Paracelsus-Elena Clinic, Kassel, Germany
}

\begin{abstract}
Parkinson's disease (PD) is frequently compounded by neruropsychiatric complications, increasing disability. The combined effect of motor and mental status on care-dependency in PD outpatients is not well characterized. We conducted a cross-sectional study of 1449 PD outpatients. The assessment comprised the Montgomery-Asberg Depression Rating Scale (MADRS) and the diagnostic criteria for dementia. PD severity and treatment complications were rated using Hoehn and Yahr staging and the Unified Parkinson's Disease Rating Scale (UPDRS) IV. The acknowledged level of care-dependency was documented. Caredependency was present in $18.3 \%$ of all patients. A total of $13.9 \%$ had dementia, $18.8 \%$ had depression, and $14.3 \%$ had both. Regression analyses revealed increasing effects of age, PD duration, and PD severity on care-dependency in all three mental-disorder subgroups with the strongest effects in patients with depression only. Depressed patients with antidepressive treatment still had significantly higher PD severity, higher MADRS and UPDRS-IV scores but were not more likely to be care-dependent than non-depressed patients. Older age, longer duration and increased severity of PD contribute to care-dependency in patients with untreated depression. Treatment of depression is associated with lower rates of caredependency.
\end{abstract}

Keywords: Depression, Parkinson's disease, Dementia, Care

\section{Introduction}

Parkinson's disease (PD) is a common neurodegenerative disorder among the elderly, characterized by progressing motor impairment leading to increasing physical disability [1]. It can also be accompanied by neuropsychiatric complications such as dementia or depression, aggravating the course of the disease in $25-50 \%$ of all patients $[2,3]$. Both motor and neuropsychiatric aspects increase risk of care-dependency. However, while the effect of 
neuropsychiatric symptoms on care-dependency has been evaluated for patients with dementia without PD [4], the relationships among motor features, neuropsychiatric complications, and care-dependency in outpatients with PD have been neglected. Recent socioeconomic studies on PD patients have identified disease severity, dementia, and depression as substantial cost drivers in the course of the disease but have not investigated the direct links between these factors and care-dependency $[5,6]$.

Recently, we published results from a large representative sample of 1449 PD outpatients (German Study on the Epidemiology of Parkinson's Disease with Dementia, GEPAD), providing an overview of the associations between neuropsychiatric symptoms in PD and care-dependency [7]. In the light of its amenability to various intervention strategies [8], we focus on the role of depression and its medicamentous treatment in the occurrence of caredependency in PD outpatients with and without dementia in this paper.

\section{Methods}

\subsection{Study design}

The study design and materials of GEPAD have been described previously in greater detail [9]. In summary, GEPAD was a cross-sectional study involving 1449 outpatients with PD, randomly selected and clinically assessed by a nationwide representative sample of 315 office-based neurologists on a single study day. The assessment comprised standardized instruments including the Montgomery-Asberg Depression Rating Scale (MADRS) and the diagnostic DSM-IV criteria for dementia, obtained in a structured clinical interview [10,11]. Based on the validation studies by Leentjens et al. [12], our study criteria for depression were either a MADRS total score $\geq 14$ and/or current treatment with antidepressants. In this paper, we distinguish between patients with depression (study criteria for depression fulfilled, but no dementia present), dementia (study criteria for dementia met but not for depression), or both (study criteria for depression as well as DSM-IV criteria met). The physicians recorded the current treatment status. The severity of PD was rated according to the Hoehn and Yahr staging (HY) scale. Complications in PD treatment were documented using the Unified Parkinson's Disease Rating scale (UPDRS) IV. For each patient, the physician noted the officially acknowledged level of care-dependency. In Germany, a patient's level of caredependency is determined by an expert member of the Medical Service of the Central Association of Health Insurance Funds. It is defined using one of three severity grades: one = substantially care-dependent (average daily need for assistance at least $90 \mathrm{~min}$, of which at least 45 min account for personal care such as bathing, feeding, assisting with toileting); two $=$ severely care-dependent (average daily need for assistance at least $180 \mathrm{~min}$, of which at least $120 \mathrm{~min}$ account for personal care); and three = most severely care-dependent (average daily need for assistance at least $300 \mathrm{~min}$, of which at least $240 \mathrm{~min}$ account for personal care, and nocturnal assistance also becomes necessary). In this paper, care-dependency is defined as the presence of at least level one. In the absence of an officially acknowledged level of care-dependency, the physicians were also asked to state whether the patient nonetheless met criteria for care-dependency (but application pending or not made). In this paper, if not otherwise indicated, analyses on care-dependency only include patients with an officially acknowledged level.

\subsection{Statistics}


All scores (summary-scores and subscores) were calculated according to the corresponding scoring algorithms. Associations with 95\% confidence intervals (CIs) of treatment status and PD severity and care-dependency were analyzed by regression models among patients with and without a current depression symptomatology. Logistic regression models were applied for categorical data and linear regression models for continuously distributed data. The predictive value of age, duration of PD and PD severity for care-dependency was investigated by considering the area under the receiver operating characteristics curve (AUC). Statistical inference was based on a significance level of 5\%. For standard errors, $\mathrm{CI}$, and $/ \mathrm{P} /$ values, the Huber-White sandwich estimator was implemented to take the clustered sampling design of the study into account [13]. For the detection of differences in metric and nonparametric data, we used the t-test and the Mann-Whitney test, respectively.

\subsection{Ethics}

The study was approved by the local Ethics Committee (August 11, 2005, No. EK140082005). Written informed consent was obtained from all participating patients or their caregivers.

\section{Results}

\subsection{Study population}

The majority of patients was male $(60.5 \%)$ and had a mean age of $70.7 \pm 8.4$ years, with a mean PD duration of 5.55 .1 years. According to the HY stages, $44.2 \%$ of all patients had mild PD (HY I + II), 38.7\% had moderate PD (HY III), and 17.1\% suffered from severe PD $($ HY IV + V). Regarding mental status, $13.9 \%$ had dementia, $18.8 \%$ met study criteria for depression (of which $7.3 \%$ scored MADRS $\leq 13$ but were currently treated with antidepressants), and $14.3 \%$ suffered concurrently from both disorders (i. e. dementia total: $28.2 \%$, depression total: $33.1 \%$ ). At the time of study conduction, $24.5 \%$ of all demented patients received antidementia treatment (acetylcholine esterase inhibitors, ChE-I).

A level of care-dependency was acknowledged for $18.3 \%$ of all patients $(n=266$ of 1449), of which the majority either had level one $(51.9 \%, n=138)$ or level two $(43.2 \%, n=115)$, respectively. Thirteen patients $(4.9 \%)$ had level three. One-hundred twenty-one $(8.5 \%)$ patients had no officially acknowledged level of care-dependency but met criteria for caredependency according to their attending clinicians. For 22 patients, information on caredependency status was not available to the physician; however, they did not differ from careindependent patients (i.e., no care-dependency level acknowledged and criteria not met) regarding age $(\mathrm{P}=0.951), \mathrm{HY}$-staging $(\mathrm{P}=0.311)$, or frequency of dementia $(\mathrm{P}=0.109)$ or depression $(\mathrm{P}=0.250)$.

Care-dependency occurred more frequently in patients aged $\geq 76$ years $(43.3 \%)$ than in patients at the age of $66-75(23.7 \%)$ or $\leq 65(13.9 \%)$, respectively. The mean $( \pm S D)$ MADRS score increased significantly with advancing levels of care-dependency, ranging from $7.7 \pm$ 6.8 ("none") to $13.1 \pm 8.3$ ("one"), $14.3 \pm 9.6$ ("two") and $18.5 \pm 11.3$ ("three", total group differences $\mathrm{P}<0.001$ ).

\subsection{Predictors for care-dependency}


We used a multiple stepwise logistic regression model to calculate the effect of age, PD duration, and PD severity on caredependency. We first entered age, added PD duration (step two), and finally entered PD severity (step three). Results are displayed in Fig. 1.

In all three mental-disorder subgroups, the stepwise entry of the predictors substantially increased the AUCs. The largest AUCs were seen in patients with depression (AUCs: 0.681, 0.739 , and 0.818 ), and the smaller in patients with dementia (AUCs: $0.603,0.687$, and 0.797 ) or both disorders combined (AUCs: 0.616, 0.642, and 0.782).

\subsection{Depression treatment and care-dependency}

Table 1 shows the associations among care-dependency, PD severity, PD treatment complications (UPDRS-IV), and depression, stratified by depression treatment status. Regardless of whether they were treated for depression or not, patients with a MADRS score $\geq 14$ had more severe PD, experienced more treatment complications, and were more frequently care-dependent than patients with a MADRS score $\leq 13$. Among patients below this cut-off for depression, patients who underwent antidepressive treatment were not more care-dependent $(\mathrm{P}=0.794)$ than the non-depressed $\mathrm{PD}$ group, although the former had higher HY stages $(\mathrm{P}<0.05)$, more treatment complications $(\mathrm{P}<0.05)$, and higher MADRS scores $(\mathrm{P}$ $<0.01)$. In terms of ORs, these patients had an insignificant $\mathrm{OR}$ for care-dependency $(\mathrm{OR}=$ 1.1, 95\% CI: 0.66-1.73) compared to non-depressed patients. This lack of significance still held true after adjustment for dementia (18.8\% vs. $23.9 \%$, OR=1.1, 95\% CI: $0.68-1.81)$. These effects also applied to patients without an officially acknowledged level of caredependency but who met the criteria for care-dependency according to the clinician $(\mathrm{OR}=1.0$, 95\% CI: 0.51-2.08 and OR $=1.1,95 \%$ CI: 0.52-2.14 after adjustment for dementia).

\section{Discussion}

In this paper, we analyzed the associations among PD, neuropsychiatric complications, and the degree of care-dependency, based on a large and representative sample of outpatients in Germany. According to the study criteria for dementia and depression, a large proportion of patients suffered from at least one of the disorders (47\%). About one in three patients $(33.1 \%)$ met the study criteria for depression.

Two important findings emerged from this work. First, in all three mental-disorder subgroups (depression, dementia, or both) age, duration of PD, and PD severity were clearly associated with care-dependency. PD severity contributed most to care-dependency, considerably increasing the AUCs at final entry in each subgroup. In patients with depression (but no dementia), the effects turned out to be strongest, revealing the largest AUCs when stepwise entering the variables considered. Thus, in these patients, care-dependency is driven more by these variables - especially age - than in patients with dementia (with and without concomitant depression).

Second, successfully treated (with antidepressants) subsyndromal patients (i.e., scoring below the MADRS cut-off for major depression while taking antidepressants) still experienced more severe motor impairments, more PD treatment complications, and higher MADRS scores than non-depressed patients. However, they were not more frequently care-dependent. This outcome was largely independent from the concurrent presence of dementia. In patients with dementia, the rates of care-dependency were increased overall, but the proportions and level of significance remained the same. 
Our data underscore that dementia and depression are important contributors to the overall disease burden in PD, as previously reported $[5,6,14]$. In addition, the GEPAD results highlight the substantial effect of depression on care-dependency in PD, suggesting that drug therapy for depression in PD with and without dementia is always beneficial because it reduces the personal burden of the patients.

Our findings, however, should be interpreted cautiously because of a number of methodological limitations. First, it is important to note that the acknowledgment of caredependency in Germany is mainly based on a person's incapacity to perform basic physical actions. Incapacities that result from neuropsychiatric complications and that usually increase the need for time-consuming assistance in everyday life are often taken inadequately into account. They lead, however, to a substantial degree of disability, especially for patients with mild to moderate dementia. Thus, the officially acknowledged care-dependency in our sample probably underestimates the real dependency on assistance, which relatives usually provide. Second, our results might be biased by the use of a screening tool for depression only instead of a comprehensive interview; however, because of feasibility concerns, we wanted to keep the assessment simple for the participating physicians. We are therefore unable to provide more sophisticated data on the type of depression or on previous episodes of depressive disorders. By the same token, we have not implemented a diagnostic instrument for the assessment of anxiety disorders, which are a frequent accompaniment of depression, and can equally contribute to care-dependency. Third, it should be noted that our analyses of associations between PD severity and CDP are limited by the use of the coarse-grained HYstaging instead of more sophisticated analyses based upon the UPDRS motor subscale. However, the latter one could not be implemented to the study protocol for logistical reasons, as it would have considerably prolonged the duration of assessment. Thus, to keep the study feasible for the participating office-based physicians (and their patients) during their daily routine care, we decided to omit the UPDRS III. By the same token, we lack further details regarding the patients' dementia status (e. g. duration, age at onset), as well as more detailed data about further neuropsychiatric conditions that might also contribute to care-dependency, such as anxiety disorders or psychotic syndromes. Similarly, the comprehensive documentation of these conditions would have required a considerably extended assessment. Finally, it should be considered that the prescription of antidepressants is not always a valid indicator for depression because these medications can also be prescribed for other medical conditions such as sleep disorders. However, it is noteworthy that in the GEPAD sample, the corresponding subgroup of patients featured characteristics that are rather typical for subsyndromal patients but not for non-depressed patients (i.e., still significantly higher MADRS scores, more treatment complications and motor impairments) [15]. In any case, more detailed studies are certainly needed to develop a deeper understanding of PD, neuropsychiatric symptoms, care-dependency, and mediating factors. 

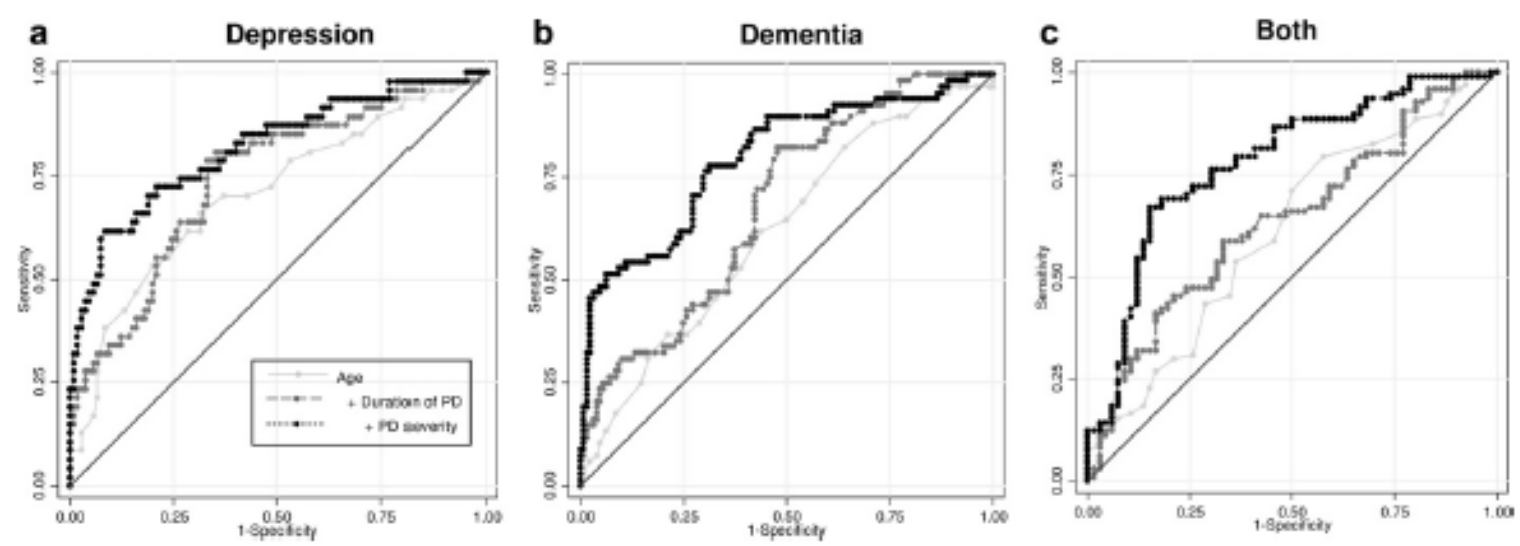

Fig 1 Impact of age, PD duration and PD severityon care dependency in patients with depressian and/ar dementin, estimated by a stepwise regression analysis by first entering age (step 1), then adding duration of PD (step 2) and PD severity (step 3)-

Table 1

PD severity and care-dependency among treated and untreated patients with and without current depression symptomalogy (MADRS $\geq 14)$.

\begin{tabular}{|c|c|c|c|c|c|c|}
\hline & \multicolumn{3}{|c|}{ MADRS $\geq 14$} & \multicolumn{3}{|c|}{ MADRS $\leq 13$} \\
\hline & Untreated & Trexted & P-value & Untreated & Trexted & P-value \\
\hline \multicolumn{7}{|l|}{ PD severity, $q$} \\
\hline Mild (HY I+II) & 230 & 327 & $c 005$ & 52.9 & 429 & sos \\
\hline $\begin{array}{l}\text { Severe } \\
(H Y N+V)\end{array}$ & 310 & 23.5 & $<005$ & 11.7 & 13.7 & $n s$ \\
\hline MADRS scre, mexl + sd & $17.3=40$ & $197 \pm 46$ & $<001$ & $49 \pm 36$ & $69 \pm 3.6$ & 2001 \\
\hline Mild (Level 1) & 153 & 158 & nx & 6.5 & $10 \mathrm{~s}$ & ne \\
\hline Moderate (level 2) & 14.1 & 121 & ns. & 6.1 & 30 & $n=$ \\
\hline Severe (Level 3) & 16 & 1.8 & $n s$ & 0.4 & ao & - \\
\hline Any & $31 \mathrm{~A}$ & 297 & $\mathrm{~ns}$ & 13.0 & 13.8 & $\mathrm{~ns}$ \\
\hline
\end{tabular}

HY: Hoehn and Yahr staging; treated/untreated - antidepressants; n.s-not significant.

\section{Conflicts of interest}

Oliver Riedel: None.

Richard Dodel: Professor Richard Dodel has received honoraria, travel grants and research grants from several pharmaceutical companies.

G. Deuschl: Stock Ownership in medically-related fields: none, Consultancies: none, Partnerships: none, Honoraria: Medtronic, Lundbeck, Teva, Grants: German Research Council, German Ministry of Education and Research, Medtronic. Employment: Government employee.

Royalties: Thieme publisher.

J. Klotsche: None.

H. Förstl: HF has received honoraria for presentations and advisory boards (Eisai, Eli-Lilly, Janssen-Cilag, Merz, Novartis, Pfizer a. o.).

I. Heuser: None.

W. Oertel: Professor Oertel has received unrestricted educational grants for clinical research projects by Novartis.

H. Reichmann: Professor Reichmann was acting on Advisory Boards and gave lectures and received research grants from Abbott, Bayer Health Care, Boehringer/Ingelheim, Cephalon, Desitin, GSK, Merck-Serono, Novartis, Orion, Pfizer, TEVA/Lundbeck, UCB Pharma, and Valeant. Peter Riederer: None.

C. Trenkwalder: Professor Claudia Trenkwalder was acting on advisory boards for Novartis. H.-U. Wittchen: H.-U. Wittchen has received an unrestricted educational grant by Novartis Pharma (Germany) for the GEPAD study. 


\section{Financial disclosure}

None.

\section{Acknowledgments}

The GEPAD study was supported through an unrestricted educational grant through Novartis Pharma GmbH (Nuremberg, Germany). The GEPAD study group wishes to thank all cooperating physicians and patients for their participation.

\section{References}

[1] Klockgether T. Parkinson's disease: clinical aspects. Cell Tissue Res 2004;318: 115-20. [2] Aarsland D, Zaccai J, Brayne C. A systematic review of prevalence studies of dementia in Parkinson's disease. Mov Disord 2005;20:1255-63.

[3] Reijnders J, Ehrt U, Weber W, Aarsland D, Leentjens A. A systematic review of prevalence studies of depression in Parkinson's disease. Mov Disord 2008;23: 183-9.

[4] Gräßel E, Donath C, Lauterberg J, Haag C, Neubauer S. Dementia patients and levels of care: do symptoms of the illness affect the grading? Gesundheitswesen 2008;70:129-36.

[5] Ehret R, Balzer-Geldsetzer M, Reese JP, Dodel I, Becker E, Christopher A, et al. Direct costs for Parkinson's treatment in private neurology practices in Berlin. Nervenarzt 2009;80:452-8.

[6] Winter Y, Balzer-Geldsetzer M, Spottke A, Reese JP, Baum E, Klotsche J, et al. Longitudinal study of the socioeconomic burden of Parkinson's disease in Germany. Eur J Neurol 2010;17:1156-63.

[7] Riedel O, Dodel R, Deuschl G, Förstl H, Henn F, Heuser I, et al. Dementia and depression determine care dependency in Parkinson's disease. Nervenarzt 2011;82:1012-9.

[8] Okun M, Watts R. Depression associated with Parkinson's disease e clinical features and treatment. Neurology 2002;58:S63-70.

[9] Riedel O, Klotsche J, Spottke A, Deuschl G, Foerstl H, Henn F, et al. Frequency of dementia, depression and other neuropsychiatric symptoms in 1,449 outpatients with Parkinson's disease. J Neurol 2010;257:1073-82.

[10] American Psychiatric Association. Diagnostic and statistical manual of mental disorders - text revision. 4th ed. Washington, DC: American Psychiatric Association; 2000.

[11] Montgomery S, Asberg M. A new depression scale, designed to be sensitive to change.

Br J Psychiatry 1979;134:382-9.

[12] Leentjens A, Verhey F, Lousberg R, Spitsbergen H, Wilmink F. The validity of the Hamilton and Montgomery-Asberg depression rating scales as screening and diagnostic tools for depression in Parkinson's disease. Int J Geriatr Psychiatry 2000;15:644-69.

[13] Royall RM. Model robust confidence intervals using maximum likelihood estimators. Int Stat Rev 1986;54:221-6.

[14] Winter Y, von Campenhausen S, Brozova H, Skoupa J, Reese J, Bötzel K, et al. Costs of Parkinson's disease in Eastern Europe: a Czech cohort study. Parkinsonism Relat Disord 2010;16:51-6.

[15] Riedel O, Heuser I, Klotsche J, Dodel R, Wittchen H-U. Occurrence risk and structure of depression in Parkinson disease with and without dementia: results from the GEPAD study. $\mathrm{J}$ Geriatr Psychiatry Neurol 2010;23:27-34. 